\title{
Interactive involvement of hippocampal cAMP/PKA and cyclooxygenase-2 signaling pathways in spatial learning in the Morris water maze
}

\author{
Kaveh Tabrizian ${ }^{1}$, Mahmoud Hashemzaei ${ }^{1}$, Ali Akbar Nasiri², Sheyda Najafi ${ }^{3}$, Fatemeh Amelinia ${ }^{4}$, Mehdi Sanati ${ }^{5}$, \\ Farzaneh Shamshirgaran ${ }^{4}$, Sahar Fanoudi ${ }^{6}$ \\ ${ }^{1}$ Department of Pharmacology and Toxicology, Faculty of Pharmacy, Zabol University of Medical Sciences, Zabol, ${ }^{2}$ Department \\ of Anesthesiology, Faculty of Medicine, Zabol University of Medical Sciences, Zabol, ${ }^{3}$ Pharmaceutical Care Department, College of Pharmacy, \\ Tehran University of Medical Sciences, Tehran, ${ }^{4}$ Students Research Committee, Faculty of Pharmacy, Zabol University of Medical \\ Sciences, Zabol, ${ }^{5}$ Department of Pharmacology and Toxicology, Faculty of Pharmacy, Tehran University of Medical Sciences, Tehran, \\ ${ }^{6}$ Department of Pharmacology, Faculty of Medicine, Mashhad University of Medical Sciences, Mashhad, Iran
}

\begin{abstract}
Introduction: Accumulated evidence shows that the CAMP-PKA signaling pathway plays a key role in memory functions. Cyclooxygenase-2, a critical player in neuroinflammation, has been confirmed in the pathogenesis of neurodegenerative diseases. This study is aimed to assess the effect of the interaction of CAMP-PKA and cyclooxygenase pathways on spatial memory acquisition in animal models.

Material and methods: In the present study, the effects of the four-day bilateral intra-hippocampal infusions of H-89 as a protein kinase All inhibitor $(10 \mu \mathrm{M} / \mathrm{side})$, celecoxib $(0.1 \mathrm{M} / \mathrm{side})$ as a selective cyclooxygenase-2 inhibitor, celecoxib/H-89 and bucladesine (10 $\mathrm{\mu M} / \mathrm{side}) / c e l e c o x i b / \mathrm{H}-89$ on spatial memory acquisition in the Morris water maze were investigated. Control animals received bilateral intra-hippocampal infusions of dimethyl sulfoxide. Rats were trained for 4 days; each day included one block of four trials. Post-training probe trial tests were performed on day five.

Results: A bilateral intra-hippocampal infusion of $\mathrm{H}-89$ and celecoxib led to a significant impairment in spatial learning compared to the controls through a notable decrease in escape latency and traveled distance. But, combination treatment of animals with celecoxib/H-89 and bucladesine/celecoxib/H-89 could considerably reverse celecoxib and H-89-induced spatial memory acquisition impairments in the Morris water maze.

Conclusions: These results indicate the probable regulatory effects of CAMP/PKA and cyclooxygenase-2 signaling pathways on spatial memory acquisition in the Morris water maze.
\end{abstract}

Key words: COX-2, cAMP/PKA, Morris water maze, spatial memory acquisition, hippocampus.

\section{Introduction}

The hippocampus and the adjacent cortex are broadly acknowledged structures for memory processes [31]. A considerable body of evidence about hippocampal lesion-induced learning and memory deficits has confirmed the vital role of the hippocampus as represented by synaptic plasticity in formation of memory [1,31]. 
Recent studies have supported the essential function of CAMP/PKA signaling pathway in the final phases of memory consolidation [12]. PKA-induced CREB phosphorylation in the hippocampus accompanied by sensitivity to inhibitors of gene transcription and protein synthesis during PKA active periods, suggest the important role of this signaling pathway in long-term memory formation [12]. Moreover, previous published data on intra-hippocampal infusion of $\mathrm{H}-89$ as a selective PKA II inhibitor and dibutyryl cyclic AMP (bucladesine) as a membrane permeable selective activator of PKA into the CA1 region of the hippocampus, plus behavioral and immuno-histochemical findings have suggested the importance of PKA in spatial memory retention and its regulation of cholinergic gene expression $[2,27,30]$. Several studies indicated that PKA signaling pathway inhibition led to learning and memory deficiency $[8,21]$ and enhancement of apoptosis and autophagy [14]. Cyclooxygenase (COX) is the key enzyme that converts arachidonic acid to prostaglandins [33]. Two unique cyclooxygenase genes (COX-1 and COX-2) and a putative third isoform (COX-3) as a splice variant of the COX-1 transcript have been identified [13]. Of the three isozymes of COX that have been known, COX-2 has attracted a growing attention because of its involvement in cognitive functions and inflammatory processes [33]. Investigations of the inflammatory signaling pathways to determine potential interaction of COX with learning and memory have demonstrated that celecoxib as a COX-2 selective inhibitor could impair the spatial memory acquisition and retention in rodents $[1,26]$. COX-2 protein has been recognized particularly in the cortex, hippocampus, amygdala and dorsal horn of the spinal cord of both rodent's and human central nervous system (CNS) [13].

On the other hand, glutamate is a major excitatory neurotransmitter in the brain involved in numerous brain functions, such as synaptic plasticity, learning and memory [11]. It is important to note that the colocalization of COX-2 protein with glutaminergic neurons in dentate gyrus and CA1-CA3 pyramidal layers of the hippocampus confirms the link of neuronal COX-2 expression with excitatory activity in CNS [13].

In the present study, the intra-hippocampal effects of $\mathrm{H}-89$, celecoxib, and also interactive effects of celecoxib/H-89 and bucladesine/celecoxib/H-89 on spatial learning acquisition in the Morris water maze (MWM) were investigated.

\section{Material and methods}

\section{Animals}

Male Wistar rats (180-220 g, purchased from the Faculty of Pharmacy, Zabol University of Medical Sciences, were housed at $25 \pm 2^{\circ} \mathrm{C}$ with a 12-h light/dark cycle and free access to food and water. All animal procedures were done in accordance with the Ethical Committee guidelines for the care and use of laboratory animals of the Zabol University of Medical Sciences.

\section{Drugs}

Celecoxib, H-89 [n-(2-aminoethyl)-5-isoquinoline-sulfonamide] and bucladesine (dibutyryl CAMP, DB-CAMP) were purchased from Sigma (St. Louis, $\mathrm{MO}$ ) and dissolved in DMSO (dimethyl sulfoxide) to obtain desirable concentrations $(0.1 \mathrm{M}, 10 \mu \mathrm{M}$ and $10 \mu \mathrm{M}$, respectively). Ketamine (100 mg/kg, i.p.) and xylazine $(25 \mathrm{mg} / \mathrm{kg}$, i.p.) were used for induction of anesthesia for stereotaxic surgery.

\section{Surgery and drug infusions}

After induction of anesthesia, bilateral cannulation in the CA1 region of the hippocampus was performed using a stereotaxic instrument according to Paxinos and Watsons atlas of the rat brain $[9,24]$. Bilateral intra-hippocampal infusions of $1 \mu \mathrm{l} / \mathrm{side}$ of celecoxib (0.1 M), H-89 $(10 \mu \mathrm{M})$, bucladesine $(10 \mu \mathrm{M})$ and DMSO were carried out through the guide cannula. All infusions were done 30 minutes before training each day. In combination treatments, celecoxib + $\mathrm{H}-89$ or bucladesine + celecoxib $+\mathrm{H}-89$, the infusion procedure involved a 5 minutes' interval between each microinjection.

\section{Behavioral trainings and learning assessments}

Four-day training (including one block of four trials) in MWM was performed according to our previous studies $[16,27]$. Spatial acquisition was assessed through measuring escape latency, traveled distance, and swimming speed by the EthoVision video tracking system (Noldus Information Technology, Wageningen, the Netherlands) as described previously [3,26-28,30]. Post-training probe trial tests were performed on day 5 to examine the time spent in the target quadrant (the quadrant included a hidden platform during training trials). In the probe trial test, the hidden platform was removed and animals were allowed to swim freely for 90 seconds. 


\section{Experiments}

\section{Experiment 1}

In the first experiment, the effects of bilateral intra-hippocampal infusions of $\mathrm{H}-89(10 \mu \mathrm{M} / \mathrm{side})$ and celecoxib $(0.1 \mathrm{M} / \mathrm{side})$ in a volume of $1 \mu \mathrm{l} / \mathrm{side}$ on spatial learning acquisition were explored. Control animals received $1 \mu \mathrm{l} /$ side DMSO.

\section{Experiment 2}

The purpose of this experiment was to examine (i): the interactive effects of celecoxib $(0.1 \mathrm{M} /$ side) and $\mathrm{H}-89(10 \mu \mathrm{M} / \mathrm{side})$, and (ii): effects of interaction of bucladesine, celecoxib and $\mathrm{H}-89$ on spatial learning acquisition in MWM. The animals received bilaterally combination doses of bucladesine $(10 \mu \mathrm{M} /$ side), celecoxib $(0.1 \mathrm{M} /$ side $)$ and $\mathrm{H}-89(10 \mu \mathrm{M} / \mathrm{side})$ respectively in the CA1 region of the hippocampus. The time interval between each microinjection was 5 minutes. Post-training probe trial tests were performed in both experiments on day 5 to determine the time spent in the target quadrant.

\section{Statistical analysis}

Unpaired $t$-test, one-way analysis of variance (ANOVA) and two-way ANOVA followed by Bonferroni's post hoc test, using Graph Pad Prism 5 were used to compare the results of our behavioral studies. The statistical significance was considered at $p$ values less than 0.05 .

\section{Results}

\section{Experiment 1: Effects of celecoxib and $\mathrm{H}-89$ on spatial learning in MWM}

The results of the four-day training program in $\mathrm{H}-89(10 \mu \mathrm{M} / \mathrm{side})$ - and celecoxib (0.1 M/side)-treated animals and their related controls are presented in Figures 1 and 2. The rats receiving bilateral intra-hippocampal infusion of either $\mathrm{H}-89$ or celecoxib showed a significant increase in both traveled distance $\left({ }^{\star \star} p<0.01\right.$, Figs. $1 \mathrm{~A}$ and $\left.2 \mathrm{~A}\right)$ and escape latency $\left({ }^{* *} p<0.01\right.$, Fig. 1B and ${ }^{* * *} p<0.001$, Fig. 2B) parameters compared to control groups, while no significant differences were found in the swimming speed
A

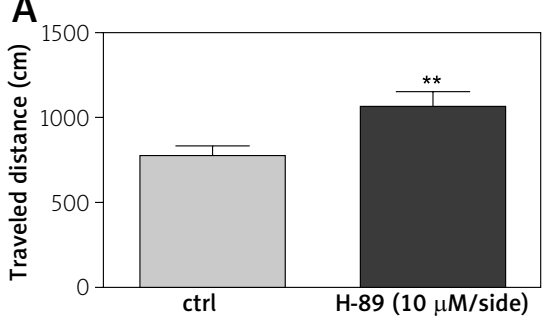

B

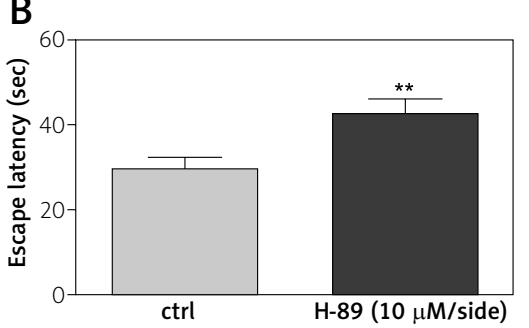

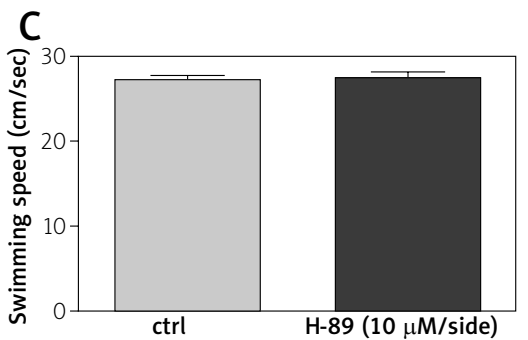

Fig. 1. An average of traveled distance (A), escape latency (B), and swimming speed (C) during four training days in the Morris water maze task in $\mathrm{H}$-89-treated animals. ${ }^{* *} p<0.01$ shows a considerable difference as compared to the control (DMSO) group. Values represent means \pm SEM of 6 animals in each group.

A

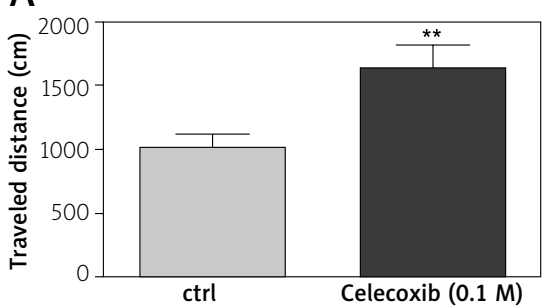

B

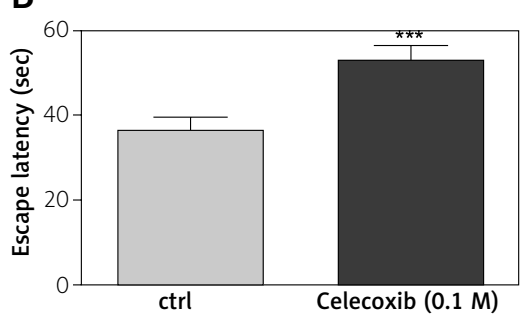

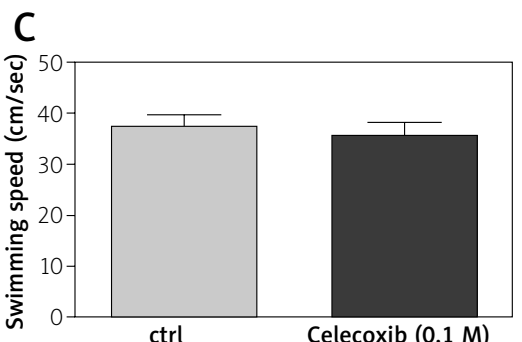

ctrl

Celecoxib (0.1 M)

Fig. 2. An average of traveled distance (A), escape latency (B) and swimming speed (C) during four training days in the Morris water maze task in celecoxib-treated animals. ${ }^{* \star} p<0.01$ and ${ }^{* \star *} p<0.001$ show a significant difference as compared to the control (DMSO) group. Values represent means \pm SEM of 6 animals in each group. 
between these animals ( $p>0.05$ ) in comparison to controls (Figs. $1 \mathrm{C}$ and $2 \mathrm{C}$ ). Considering the same training days in these treated animals, infusion of H-89 caused significant memory impairments via increasing traveled distance and escape latency at the fourth $\left({ }^{*} p<0.05\right)$ day of training (Fig. 3A and $3 B$ ) in comparison to the controls (DMSO-treated). In addition, a significant increase in the traveled distance and escape latency at the third and fourth $\left.{ }^{*} p<0.05\right)$ day of training were observed in celecoxib-treated animals in comparison to the controls (Fig. 4A and 4B).

\section{Experiment 2: Effect of co-administration of celecoxib/H-89 and bucladesine/ celecoxib/H-89 on spatial learning in MWM}

Figure 5 demonstrates the results obtained from (i): intra-hippocampal infusion of $\mathrm{H}-89(10 \mu \mathrm{M} /$ side $)$, 5 minutes after celecoxib (0.1 M/side) administration and (ii): infusion of bucladesine (10 $\mu \mathrm{M} / \mathrm{side})$, celecoxib (0.1 M/side, i.h.) and H-89 (10 $\mu \mathrm{M} /$ side, i.h.) with a time interval of 5 minutes, respectively. There were significant differences in traveled distance ( ${ }^{\#} p<0.05$, Fig. 5A) between the celecoxib/H-89 or bucladesine/celecoxib/H-89 groups compared to $\mathrm{H}$-89-treated animals during four days of training. Similarly, there was a significant increase in the traveled distance (Fig. 5A) between the celecoxib/H-89 $\left({ }^{+} p<0.05\right)$ and bucladesine/celecoxib/H-89 $\left({ }^{++} p<0.01\right)$ group compared to celecoxib-treated animals during four days of training.

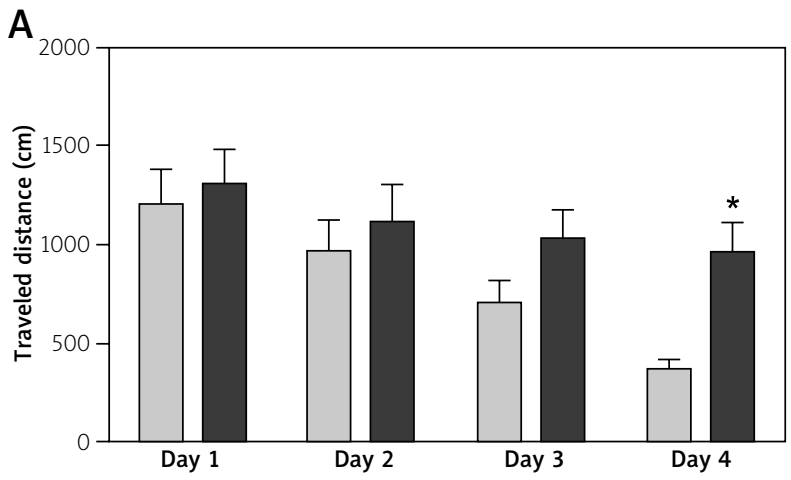

Regarding the escape latency parameter, there was a significant difference between the celecoxib/ $\mathrm{H}-89$ or bucladesine/celecoxib/H-89 groups compared to $\mathrm{H}-89$ - ( ${ }^{\#} p<0.05$, Fig. 5B) and also celecoxib ${ }^{+++} p<0.001$, Fig. 5B)-treated animals during four days of training.

Considering the swimming speed, no significant differences were found ( $p>0.05$, Fig. 5C) between control, celecoxib/H-89- and bucladesine/celecoxib/ $\mathrm{H}$-89-treated animals.

Interestingly during the same training days, there were considerable differences among celecoxib/ $\mathrm{H}-89$ or bucladesine/celecoxib/H-89 groups in the traveled distance and escape latency parameters at the fourth day of training compared to celecoxib$\left({ }^{+} p<0.05\right.$, Fig. $6 \mathrm{~A}$ and $\left.6 \mathrm{~B}\right)$ and $\mathrm{H}-89\left({ }^{\#} p<0.05\right.$, Fig. 7A and 7B)-treated groups.

\section{Evaluation of the probe trial test in $\mathrm{H}-89$, celecoxib, celecoxib/ $\mathrm{H}-89$ and bucladesine/celecoxib/ $\mathrm{H}$-89-treated animals in MWM}

Intra-hippocampal infusion of $\mathrm{H}-89$ (10 $\mu \mathrm{M}$ / side) and celecoxib (0.1 M/side) caused a significant decrease $\left({ }^{* *} p<0.001\right)$ in the time spent in the target quadrant compared to control groups. On the other hand, the time spent in the target quadrant was increased significantly in animals treated with a combination of celecoxib/H-89 or bucladesine/celecoxib/ $\mathrm{H}-89$ compared to $\mathrm{H}-89$ (\#\# $p<0.001)$ or celecoxib $\left.{ }^{+++} p<0.001\right)$-treated animals (Fig. 8).

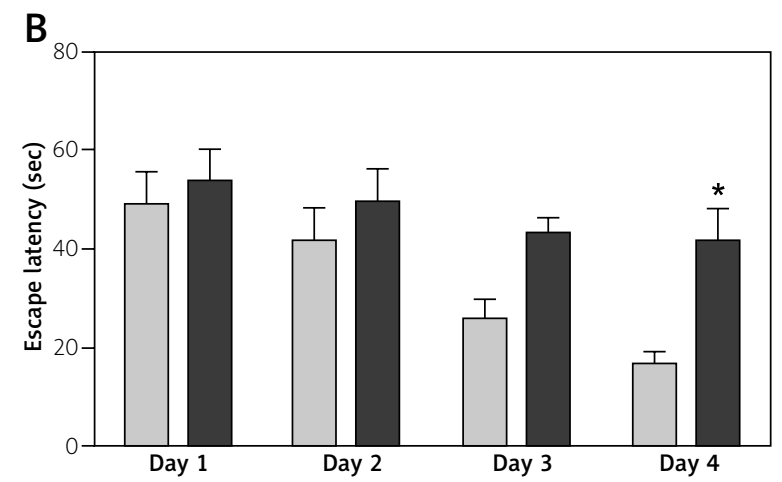

Ctrl
- H-89 (10 mM/side)

Fig. 3. Assessment of traveled distance (A) and escape latency (B) on the same training day in $\mathrm{H}$-89-treated animals. A substantial increase in the traveled distance and escape latency was observed in the $\mathrm{H}-89$ group $\left({ }^{*} p<0.05\right)$ on day 4 . Data are presented as means \pm SEM of 6 animals in each group. 
A

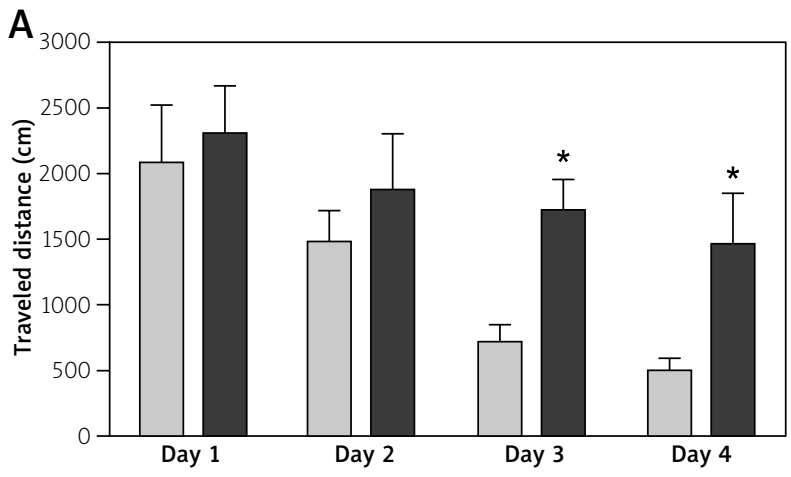

B

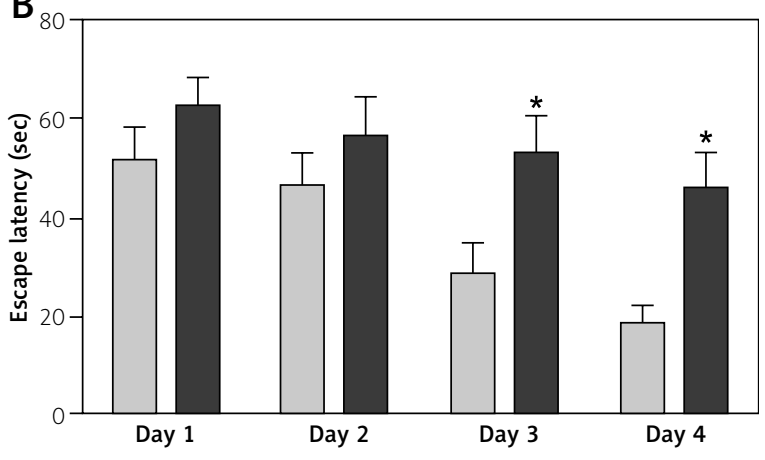

$\square$ Celecoxib $(0.1 \mathrm{M})$

Fig. 4. Evaluation of traveled distance (A) and escape latency (B) on the same training day in celecoxibtreated animals. A considerable increase in the traveled distance and escape latency was observed in the celecoxib group $\left({ }^{*} p<0.05\right)$ on days 3 and 4 . Data are presented as means \pm SEM of 6 animals in each group.

A

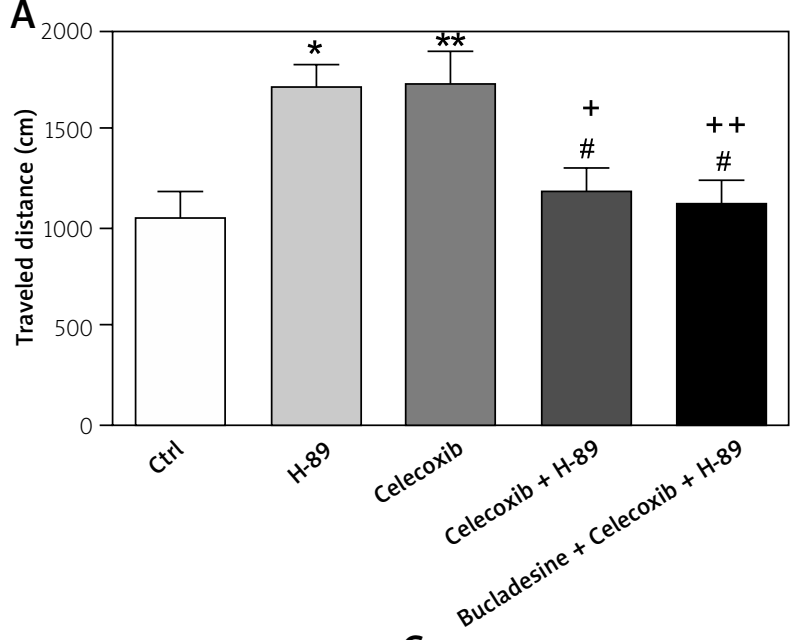

B

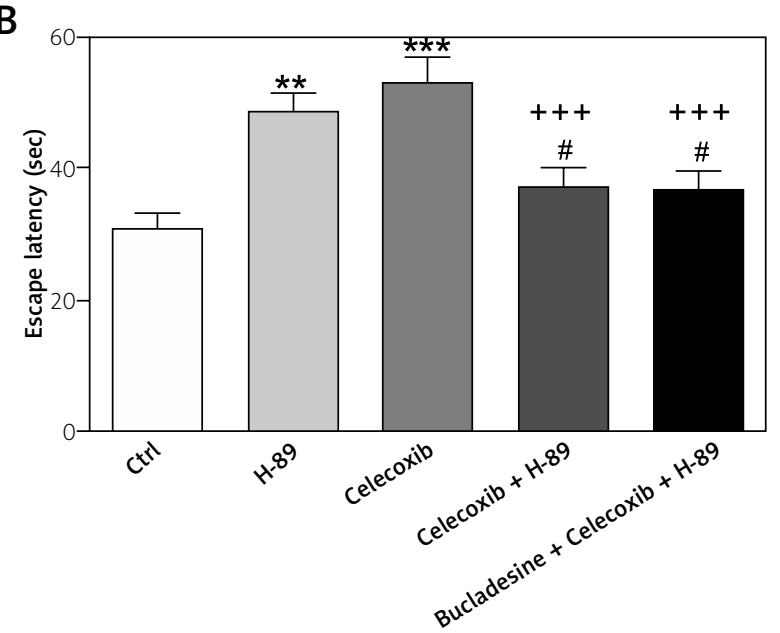

C

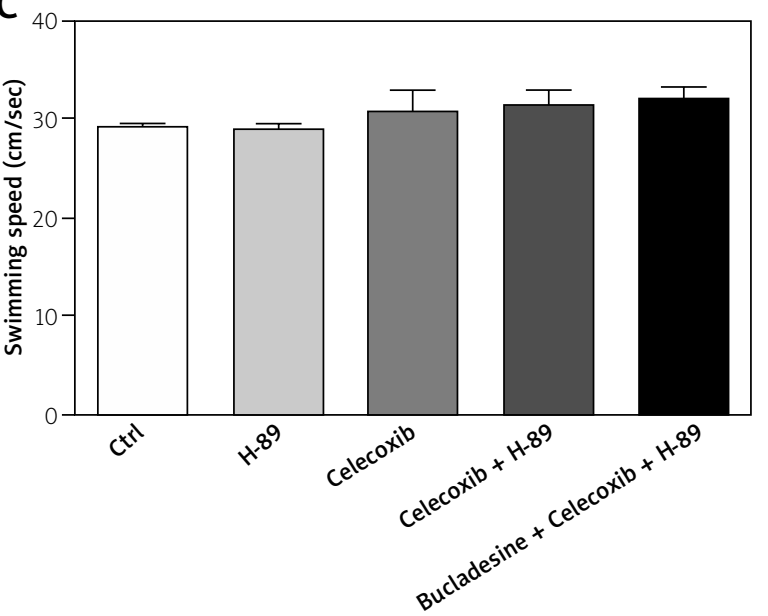

Fig. 5. An average of traveled distance (A), escape latency (B) and swimming speed (C) during four training days in the Morris water maze task for H-89-, celecoxib-, celecoxib/H-89- and bucladesine/celecoxib/ $\mathrm{H}$-89-treated animals. ${ }^{*} p<0.05,{ }^{* *} p<0.01$ and ${ }^{* * *} p<0.001$ show a notable difference as compared to the control (DMSO) group. ${ }^{\#} p<0.05$ shows a considerable difference as compared to the $\mathrm{H}$-89-treated group. ${ }^{+} p<0.05,{ }^{++} p<0.01$ and ${ }^{+++} p<0.001$ demonstrates a remarkable difference as compared to the celecoxibtreated group. Values represent means \pm SEM of 6 animals in each group. 

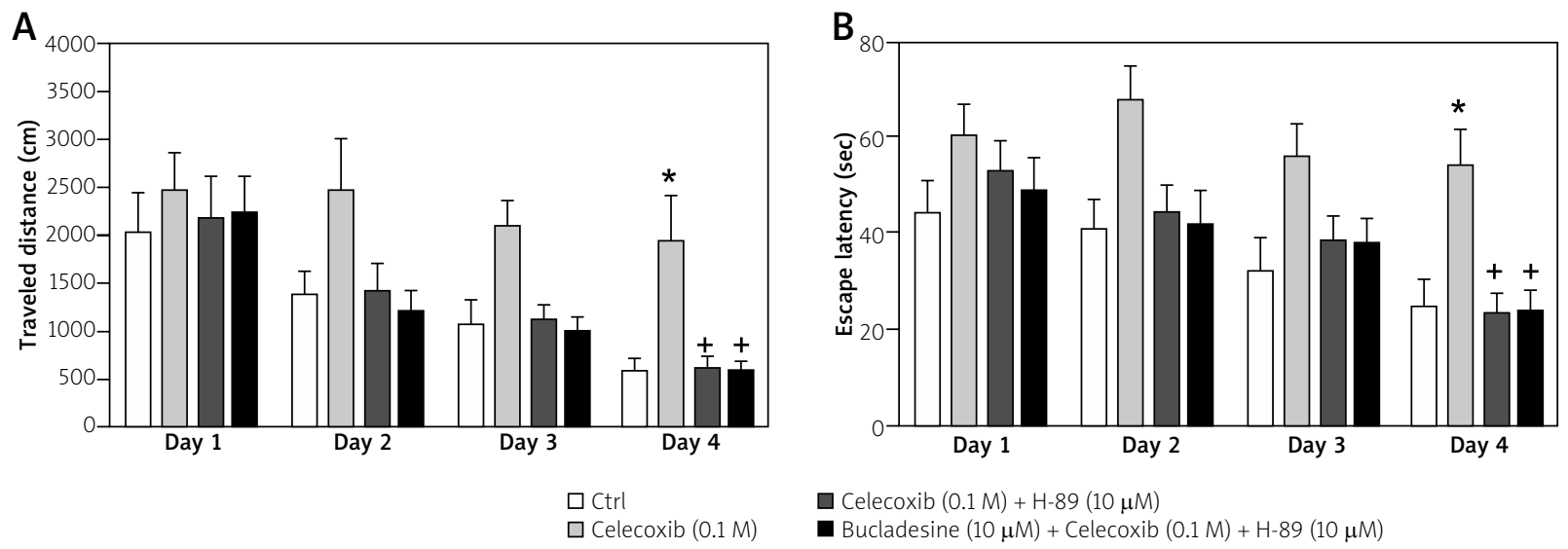

Fig. 6. Investigation of traveled distance (A) and escape latency (B) on the same training day in celecoxib-, celecoxib/H-89- and bucladesine/celecoxib/H-89-treated animals. ${ }^{*} p<0.05$, describes a considerable difference as compared to the control (DMSO) group. ${ }^{+} p<0.05$ shows a meaningful difference as compared to the celecoxib-treated group. Values represent means \pm SEM of 6 animals in each group.
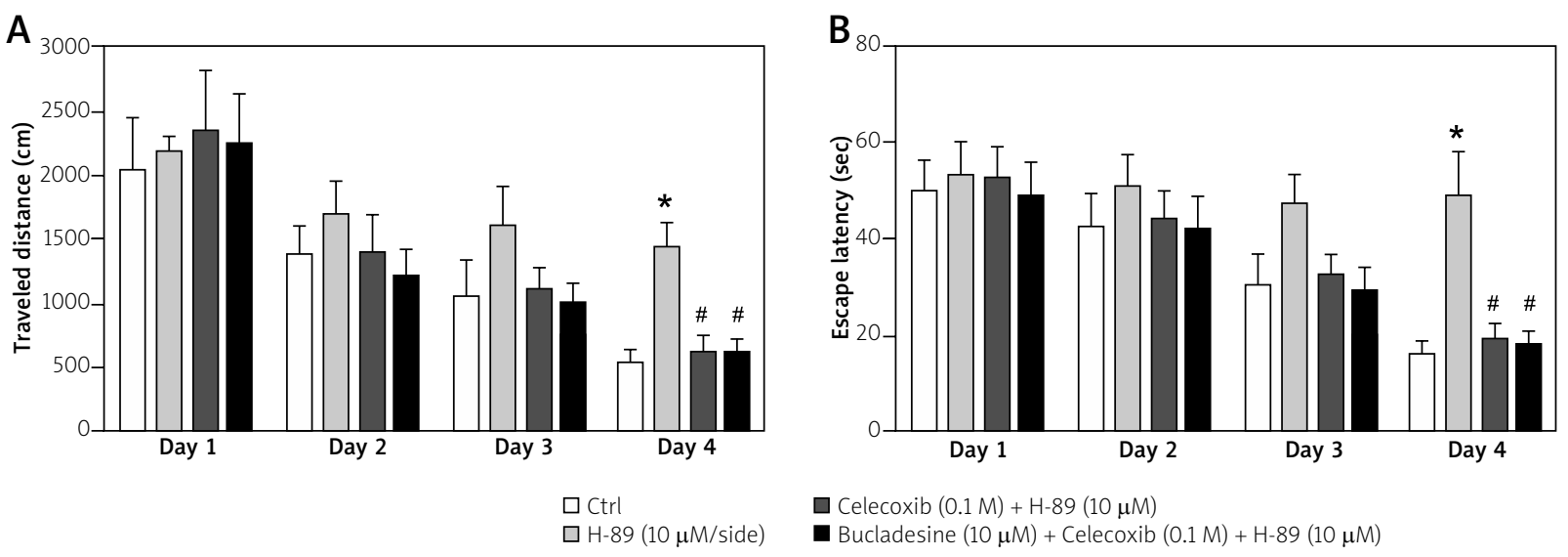

Fig. 7. Evaluation of traveled distance (A) and escape latency (B) on the same training day in $\mathrm{H}-89-$-, celecoxib/H-89- and bucladesine/celecoxib/H-89-treated animals. ${ }^{*} p<0.05$ show a meaningful difference as compared to the control (DMSO) group. ${ }^{*} p<0.05$ indicates a considerable difference as compared to the $\mathrm{H}$-89-treated group. Values represent means \pm SEM of 6 animals in each group.

\section{Discussion}

To evaluate the potential interaction of CAMP/PKA and COX-2 signaling pathways, the drugs - bucladesine, a selective activator of PKA, H-89 as a PKA II inhibitor, and celecoxib as a selective COX-2 inhibitor were used in this study. $\mathrm{H}$-89- and celecoxib-treated animals were not trained well and showed a remarkable increase in time and distance to find the hidden platform. Moreover a significant decrease in the time spent in the target quadrant was observed in comparison to control animals.

The importance of CAMP-dependent PKA in the expression of long-term potentiation, long-term depres- sion, hippocampal long-term memory, and synaptic plasticity had been reported previously [30]. Also it has been proposed that the hippocampal CAMP/PKA pathway could be activated following NMDA receptor-mediated $\mathrm{Ca}^{2+}$ influx via stimulation of adenylate cyclase activity, CAMP accumulation, and PKA and CREB phosphorylation [12].

It has been claimed that intra-hippocampal bucladesine infusion could improve spatial memory retention via synergistic interaction with nicotine as represented by significant increases in ChAT and VAChT protein expressions in the CA1 region and medial septal area of the hippocampus [2,30]. On the other hand, post training intra-hippocampal infusion 


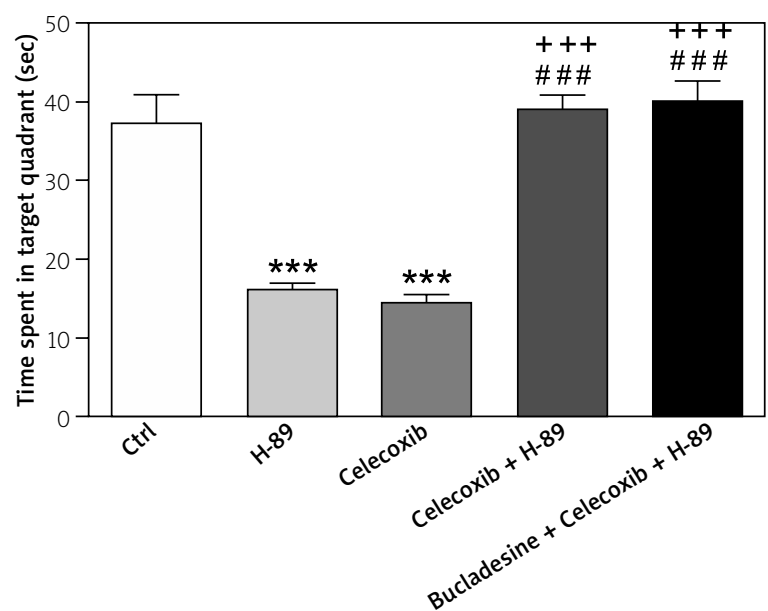

Fig. 8. Measurement of time spent in the target quadrant in the probe trial test. ${ }^{* * *} p<0.001$ shows a significant difference as compared to the control group. ${ }^{\# \# p} p<0.001$ illustrates a considerable difference as compared to the H-89-treated group. ${ }^{++} p<0.001$ displays a considerable difference as compared to the celecoxib-treated group. Values represent means \pm SEM of 6 animals in each group.

of $\mathrm{H}-89$ in our previous studies [27] could damage spatial memory retention as evidenced by the qualitative and quantitative decrease in the density of ChAT-containing cholinergic nerve terminals in the dorsal hippocampus and medial septum. Similarly, bilateral intra-hippocampal infusion of $\mathrm{H}-89$ in the present study impaired spatial learning in animals. These findings suggest the pivotal role of PKA II in regulation of cholinergic gene expression and spatial memory acquisition and retention in rats.

The key function of prostaglandin E2 (the predominant reaction product of COX-2) in neural plasticity as well as modulatory effects of prostaglandins on adrenergic, noradrenergic, and glutaminergic transmission have been investigated in previous studies. In addition, the regulatory effects of acetylcholine on COX-2 activity have suggested the interaction of the prostaglandin pathway and the cholinergic system in neuronal plasticity. However, there are some controversial findings about other physiological and pathological functions of COX-2 in the CNS [26]. It has been shown that endogenous basal levels of PGE2 production following COX-2 activity are essential for acquisition of memory and synaptic plasticity [7]. Inhibition of COX-2 suppresses glutamatergic neurotransmission and long-term potentiation in the hippocampus. These events are mostly mediated by prostaglandin E2 $[6,20]$ which consequently increases glutamate release [22]. Celecoxib is a highly selective cell permeable COX-2 inhibitor [25] and might prevent glutamate release by reducing the levels of $\mathrm{Ca}^{2+}$ entry through the Cav2.2 (N-type) and Cav2.1 (P/Q-type) $\mathrm{Ca}^{2+}$ channels that are linked to glutamate exocytosis in the nerve endings [19].

It has also been proven that bilateral infusion of $0.1 \mathrm{M} /$ side celecoxib could significantly damage spatial memory retention which persisted for 3 days, while nicotine could prevent the celecoxib-induced impairment by restoring the immunostaining pattern of COX-2 neurons in the rat dorsal hippocampus $[26,28,29]$. This study, in agreement with previous publications on spatial memory retention, demonstrates a probable role for COX-2 in spatial memory acquisition.

In contrast with our study, Yang et al. demonstrated that celecoxib treatment in diabetic rats could improve memory impairments through reduction of hippocampal COX-2 expression and increase in the BDNF-TrkB signaling pathway [34]. It has also been indicated that chronic unpredictable mild stress could increase COX2 expression and consequently learning and memory deficiency because of hippocampal CAMP/PKA-CREB-BDNF signaling activation [18]. Beneficial effects of COX2-specific inhibitors have been reported in treatment of the traumatic brain injury [10]. These findings may be obtained due to the use of COX2 inhibitors under the inflammation condition. But we investigated the effects of celecoxib in lack of inflammatory responses. Due to contradictory findings about the role of the cyclooxygenase pathway in learning and memory processes, it has been realized that different factors including the type of the experimental task, employed protocol, stage of memory formation and some pathological factors such as inflammatory conditions are involved in COX activity on memory functions.

In this work, co-administration of celecoxib/H-89 and bucladesine/celecoxib/H-89 could protect or reverse $\mathrm{H}$-89- and celecoxib-induced spatial memory acquisition deficits in MWM.

It has been reported that salicylate can decrease apoptosis and autophagy induced by $\mathrm{H}-89$ [4].

Interestingly, the protective effects of $\mathrm{H}-89$ on $A \beta$-induced memory impairment via a reduction of $\mathrm{NF \kappa B}$, (factor of neuronal death), and decrease in oxidative stress factors have been proposed [8]. Also, 
certain inflammatory cytokines have been identified which can induce COX-2 expression via an NF-kB pathway [15].

Based on previous records, NF- $\mathrm{KB}$ is involved in a number of brain functions, especially in neurodegenerative diseases and after injury. Glutamate (via both AMPA/KA and NMDA receptors) and neurotrophins are brain-specific activators of NF-KB with regards to participation in synaptic plasticity [23]. Likewise, NF- $\mathrm{KB}$ serves a role in synaptic transmission through regulation of COX-2 neuronal expression [32]. The COX-2 gene, induced by NF- $\kappa B$, plays a key function in inflammation and carcinogenesis [5].

It has been asserted that celecoxib can prohibit release of glutamate from nerve endings through reduction of voltage-dependent $\mathrm{Ca}^{2+}$ entry via a signaling mechanism including EP2 (prostaglandin E2 (PGE2) receptors) and PKA. Therefore PKA inhibitors might prevent the inhibitory effect of celecoxib on the release of glutamate [17].

To investigate the role of dibutyryl CAMP in spatial memory, we used the drug bucladesine, a selective activator of PKA. Previous studies suggested that intrahippocampal infusions of bucladesine improve spatial memory retention in male rats and interact synergistically with nicotine to improve spatial memory [30]. Also, it has been reported that significant increases in ChAT and VAChT protein expressions, as cholinergic markers, in the CA1 region and medial septal area of the hippocampus are the possible mechanisms of spatial memory improvement induced by nicotine-bucladesine combination [2].

In conclusion, the improvement of spatial memory acquisition by celecoxib/H-89 and bucladesine/ celecoxib/H-89 in the present study, suggest the probable regulatory effects of CAMP/PKA and COX-2 signaling pathways in spatial memory acquisition in MWM.

\section{Disclosure}

The authors report no conflict of interest.

\section{References}

1. Andrade C, Singh NM, Thyagarajan S, Nagaraja N, Rao NSK, Chandra JS. Possible glutamatergic and lipid signalling mechanisms in ECT-induced retrograde amnesia: experimental evidence for involvement of COX-2, and review of literature. J Psychiat Res 2008; 42: 837-850.

2. Azami K, Etminani M, Tabrizian K, Salar F, Belaran M, Hosseini A, Hosseini-Sharifabad A, Sharifzadeh M. The quantitative evalu- ation of cholinergic markers in spatial memory improvement induced by nicotine-bucladesine combination in rats. Eur J Pharmacol 2010; 636: 102-107.

3. Azami K, Tabrizian K, Hosseini R, Seyedabadi M, Shariatpanahi M, Noorbakhsh F, Kebriaeezadeh A, Ostad SN, Sharifzadeh M. Nicotine attenuates spatial learning deficits induced by sodium metavanadate. Neurotoxicology 2012; 33: 44-52.

4. Azimi L, Kachooeian M, Khodagholi F, Yans A, Heysieattalab S, Vakilzadeh G, Vosoughi N, Sanati M, Taghizadeh G, Sharifzadeh M. Protective effects of salicylate on PKA inhibitor (H-89)-induced spatial memory deficit via lessening autophagy and apoptosis in rats. Pharmacol Biochem Be 2016; 150: 158-169.

5. Cao Y, Prescott SM. Many actions of cyclooxygenase-2 in cellular dynamics and in cancer. J Cell Physiol 2002; 190: 279-286.

6. Chen C, Magee JC, Bazan NG. Cyclooxygenase-2 regulates prostaglandin E2 signaling in hippocampal long-term synaptic plasticity. J Neurophysiol 2002; 87: 2851-2857.

7. Cowley T, Fahey B, O'Mara S. COX-2, but not COX-1, activity is necessary for the induction of perforant path long-term potentiation and spatial learning in vivo. Eur J Neurosci 2008; 27 : 2999-3008.

8. Eftekharzadeh B, Ramin M, Khodagholi F, Moradi S, Tabrizian K, Sharif R, Azami K, Beyer C, Sharifzadeh M. Inhibition of PKA attenuates memory deficits induced by beta-amyloid (1-42), and decreases oxidative stress and NF-kappaB transcription factors. Behav Brain Res 2012; 226: 301-308.

9. George P, Charles W. The rat brain in stereotaxic coordinates. Qingchuan Zhuge translate (People's Medical Publishing House, Beijing, China, 2007) 1998; 32.

10. Gopez JJ, Yue H, Vasudevan R, Malik AS, Fogelsanger LN, Lewis S, Panikashvili D, Shohami E, Jansen SA, Narayan RK. Cyclooxygenase-2-specific inhibitor improves functional outcomes, provides neuroprotection, and reduces inflammation in a rat model of traumatic brain injury. Neurosurgery 2005; 56: 590-604.

11. Greenamyre JT, Porter R. Anatomy and physiology of glutamate in the CNS. Neurology 1994; 44: S7-13.

12. Guerra GP, Mello CF, Bochi GV, Pazini AM, Fachinetto R, Dutra RC, Calixto JB, Ferreira J, Rubin MA. Hippocampal PKA/CREB pathway is involved in the improvement of memory induced by spermidine in rats. Neurobiol Learn Mem 2011; 96: 324-332.

13. Hewett SJ, Bell SC, Hewett JA. Contributions of cyclooxygenase-2 to neuroplasticity and neuropathology of the central nervous system. Pharmacol Therapeut 2006; 112: 335-357.

14. Inoue H, Hase K, Segawa A, Takita T. H89 (N-[2-p-bromocinnamylamino-ethyl]-5-isoquinolinesulphonamide) induces autophagy independently of protein kinase A inhibition. Eur J Pharmacol 2013; 714: 170-177.

15. Jiang J, Dingledine R. Prostaglandin receptor EP2 in the crosshairs of anti-inflammation, anti-cancer, and neuroprotection. Trends Pharmacol Sci 2013; 34: 413-423.

16. Khorshidahmad T, Tabrizian K, Vakilzadeh G, Nikbin P, Moradi S, Hosseini-Sharifabad A, Roghani A, Naghdi N, Sharifzadeh M. Interactive effects of a protein kinase All inhibitor and testosterone on spatial learning in the Morris water maze. Behav Brain Res 2012; 228: 432-439.

17. Lin TY, Lu CW, Wang CC, Huang SK, Wang SJ. Cyclooxygenase 2 inhibitor celecoxib inhibits glutamate release by atten- 
uating the PGE2/EP2 pathway in rat cerebral cortex endings, J Pharmacol Exp Ther 2014; 351: 134-145

18. Luo Y, Kuang S, Li H, Ran D, Yang J. cAMP/PKA-CREB-BDNF signaling pathway in hippocampus mediates cyclooxygenase 2 -induced learning/memory deficits of rats subjected to chronic unpredictable mild stress. Oncotarget 2017; 8: 35558.

19. Millán C, Sánchez-Prieto J. Differential coupling of N- and P/Q-type calcium channels to glutamate exocytosis in the rat cerebra cortex. Neurosci Lett 2002; 330: 29-32.

20. Murray H, O'Connor J. A role for COX-2 and p38 mitogen activated protein kinase in long-term depression in the rat dentate gyrus in vitro. Neuropharmacology 2003; 44: 374-380.

21. Najafi S, Payandemehr B, Tabrizian K, Shariatpanahi M, Nassireslami E, Azami K, Mohammadi M, Asadi F, Roghani A, Sharifzadeh $M$. The role of nitric oxide in the PKA inhibitor induced spatial memory deficits in rat: involvement of choline acetyltransferase. Eur J Pharmacol 2013; 714: 478-485.

22. Nishiham I, Toshiaki M, Watanabe Y, Ito S, Hayaishi O. Prostaglandin E 2 stimulates glutamate release from synaptosomes of rat spinal cord. Neurosci Lett 1995; 196: 57-60.

23. O'Neill LA, Kaltschmidt C. NF-KB: a crucial transcription factor for glial and neuronal cell function. Trends Neurosci 1997; 20: 252-258.

24. Paxinos G, Watson C. The rat brain atlas. Academic Press, San Diego 1986.

25. Rall JM, Mach SA, Dash PK. Intrahippocampal infusion of a cyclooxygenase-2 inhibitor attenuates memory acquisition in rats. Brain Res 2003; 968: 273-276.

26. Sharifzadeh M, Naghdi N, Khosrovani S, Ostad SN, Sharifzadeh K, Roghani A. Post-training intrahippocampal infusion of the COX-2 inhibitor celecoxib impaired spatial memory retention in rats. Eur J Pharmacol 2005; 511: 159-166.

27. Sharifzadeh M, Sharifzadeh K, Naghdi N, Ghahremani MH, Roghani A. Posttraining intrahippocampal infusion of a protein kinase All inhibitor impairs spatial memory retention in rats. J Neurosci Res 2005; 79: 392-400.

28. Sharifzadeh M, Tavasoli M, Naghdi N, Ghanbari A, Amini M, Roghani A. Post-training intrahippocampal infusion of nicotine prevents spatial memory retention deficits induced by the cyclo-oxygenase-2-specific inhibitor celecoxib in rats. J Neurochem 2005; 95: 1078-1090.

29. Sharifzadeh M, Tavasoli M, Soodi M, Mohammadi-Eraghi S, Ghahremani MH, Roghani A. A time course analysis of cyclooxygenase-2 suggests a role in spatial memory retrieval in rats. Neurosci Res 2006; 54: 171-179.

30. Sharifzadeh M, Zamanian A-R, Gholizadeh S, Tabrizian K, Etminani M, Khalaj S, Zarrindast M-R, Roghani A. Post-training intrahippocampal infusion of nicotine-bucladesine combination causes a synergistic enhancement effect on spatial memory retention in rats. Eur J Pharmacol 2007; 562: 212-220.

31. Shrager Y, Bayley PJ, Bontempi B, Hopkins RO, Squire LR. Spatial memory and the human hippocampus. Proceedings of the National Academy of Sciences 2007; 104: 2961-2966.

32. Yamagata K, Andreasson KI, Kaufmann WE, Barnes CA, Worley PF. Expression of a mitogen-inducible cyclooxygenase in brain neurons: regulation by synaptic activity and glucocorticoids. Neuron 1993; 11: 371-386.
33. Yang $\mathrm{H}$, Chen C. Cyclooxygenase-2 in synaptic signaling. Curr Pharm Design 2008; 14: 1443

34. Yang Y, Gao L. Celecoxib Alleviates Memory Deficits by Downregulation of COX-2 Expression and Upregulation of the BDNFTrkB Signaling Pathway in a Diabetic Rat Model. J Mol Neurosci 2017; 62: 188-198. 